\title{
Automatic Calibration of Hand-Eye-Workspace and Camera Using Hand-Mounted Line Laser
}

\author{
Jwu-Sheng Hu, Member, IEEE, and Yung-Jung Chang
}

\begin{abstract}
This paper develops a novel calibration method for simultaneously calibrating the intrinsic parameters of a camera and the hand-eye-workspace relationships of an eye-to-hand system using a line laser module. Errors in the parameters of a hand-eye coordination system lead to errors in the position targeting in the control of robots. To solve these problems, the proposed method utilizes a line laser module that is mounted on the hand to project laser beams onto the working plane. As well as calibrating the system parameters, the proposed method is effective when the eye cannot see the hand and eliminates the need for a precise calibration pattern or object. The collected laser stripes in the images must satisfy nonlinear constraints at each hand pose. A closed-form solution is derived by decoupling nonlinear relationships based on the homogeneous transform and parallel plane/line constraints. A nonlinear optimization, which considers all parameters simultaneously without error propagation problem, is to refine the closed-form solution. This two-stage process can be executed automatically without manual intervention. The computer simulation and experiment verify the effectiveness of the proposed method and reveal that using a line laser is more accurate than using a single-point laser.
\end{abstract}

Index Terms-Camera calibration, hand-eye calibration, laser, machine vision.

\section{INTRODUCTION}

$\mathbf{R}$ OBOTIC hand-eye coordination systems are reliable and flexible in many industrial applications. The hand refers to a robotic manipulator and the eye generally refers to a camera. Hand-eye systems have two basic configurations, eye-in-hand and eye-to-hand, determined by the position of the camera. A camera that is mounted on the end-effector (eye-in-hand) can be maneuvered to observe a target closely. However, a static camera (eye-to-hand) has a global view and can detect scene changes more easily. Flandin et al. [1] presented cooperation between eye-in-hand and eye-to-hand to provide both advantages. Muis and Ohnishi introduced a framework for the cooperation between two manipulators, in which one hand holds a camera [2]. Position-based visual servoing depends on camera calibration

Manuscript received November 27, 2011; revised May 17, 2012; accepted July 2, 2012. Date of publication September 4, 2012; date of current version December 11, 2013. Recommended by Technical Editor Y. Sun. This work was supported in part by the National Science Council, Taiwan, under Grant NSC 99-2221-E-009-145.

J.-S. Hu is with the Department of Electrical Engineering, National ChiaoTung University, Hsinchu 310, Taiwan (e-mail: jshu@ @n.nctu.edu.tw).

Y.-J. Chang is with the Department of Electrical and Control Engineering, National Chiao-Tung University, Hsinchu 310, Taiwan, and also with the Mechanical and Systems Research Laboratories, Industrial Technology Research Institute, Hsinchu 310, Taiwan (e-mail: nuo.ece95g@nctu.edu.tw).

Color versions of one or more of the figures in this paper are available online at http://ieeexplore.ieee.org.

Digital Object Identifier 10.1109/TMECH.2012.2212717 and hand-eye calibration. Since the pose of a control object is estimated from its projection on a camera image, inaccurate camera parameters may cause a significant error in the positioning of a target. Additionally, errors in the geometrical relationship may make the controlled end-effector positions biased. Notably, such errors can be eliminated by directly observing the end-effector. However, control points on the end-effector and target can commonly not be observed simultaneously, preventing the positioning accuracy of the visual servo system from being guaranteed [3]. Therefore, increasing the accuracy of calibration remains a major challenge in robotics.

The calibration of an entire hand-eye system involves robot calibration, camera calibration, hand/eye calibration, and robot/workspace calibration. Each calibration problem has been discussed for several decades. Camera calibration requires a reference objects, which could be a 3-D object [5], a 2-D pattern [6]-[8], a 1-D bar [9], or parallel lines [10]. Assuming that the camera has been properly calibrated, many studies have focused on calibrating geometrical relationships utilizing a 3-D object [11]-[14], 2-D pattern [15], [16], nonstructured points [17]-[19], optical-flow [20], [21], or just a single point [23], [24]. Most work on the calibration of hand-eye systems is done by separating camera calibration from hand-eye calibration, causing error propagation. In many industrial applications, the camera must be recalibrated frequently. However, in an environment that cannot be easily entered by humans to place a reference object, separating the calibrations might be inefficient. Therefore, Ma developed a method that combines the two calibrations into a single process to solve this problem for an active vision system [25].

Considering the eye-in-hand and eye-to-hand configurations for visual servoing, Staniak and Zieliński [26] analyzed the effect of calibration errors on the control. The calibration of eyeto-hand configuration in which the camera is static has rarely been discussed since the transformation can be in the same form as that of eye-in-hand configuration. Most hand-eye calibration methods are applicable to either eye-in-hand or eye-to-hand configurations. Dornaika and Horaud [27] presented a formulation to solve calibration problems associated with both configurations, indicating that these two problems are identical. However, this identity can be exploited only when the robot hand can be viewed by the eye-to-hand camera. This requirement limits the flexibility of installation and the range of potential applications. An eye occasionally fails to see a hand because of various requirements. For example, to sort products on a moving conveyor, the camera is commonly placed at a distance from the arm to compensate for the image processing delay and prevent interference in the tracking of targets on a rapidly moving 
conveyor belt. In ball-catching systems, such as described by Hu et al. [28], cameras focus on the region through which the ball initially passes and may not see the arm. Sun et al. [29] developed a robot-world calibration method using a triple laser device. Despite enabling calibration of the eye-to-hand transformation when the camera cannot see the arm, their method depends on a uniquely designed triple-laser device, thus limiting the flexibility of the arrangement of the system. Furthermore, the spatial relationship between the working plane and camera must be known in advance. Recently, Park et al. [4] proposed a new robot calibration technique that involves projecting structured laser beams from the robot hand to a plane, allowing a camera to measure accurately the positions of the two projected laser spots.

This paper proposes a method for simultaneously calibrating eye-to-hand and workspace-to-robot relationships using a handmounted line laser that is projected on the working plane. The proposed method also considers the intrinsic parameters of the camera at the same time. Using a laser module is advantageous in that the proposed method can be applied when the eye does not see the hand. Restated, this method allows for a longer working range for hand-eye coordination. The robot is manipulated and the laser is projected on a plane of unknown orientations to extract a batch of light stripes from the camera images. Since the laser is mounted rigidly and the plane is fixed in each orientation, the geometrical parameters and measurements must satisfy particular nonlinear constraints and the parameter solutions can be estimated accordingly. Closed-form solution is developed by decoupling nonlinear equations into linear forms to estimate all initial values. Therefore, the proposed calibration method does not require initial guesses of unknown parameter values to be made. To ensure high accuracy, a nonlinear optimization method is implemented to refine the estimation.

This study proposes a method that uses a line laser, rather than a single-point laser, which has been adopted in previous works [30], [31]. Since a laser line stripe provides more geometrical information concerning each hand pose than a point laser, the corresponding calibration process is more efficient. The two-stage method that is proposed here can simultaneously calibrate the hand-eye-workspace relationships and the intrinsic parameters of the camera without prior information.

This paper is organized as follows. Section II defines the problem, including system models and the parameters to be calibrated. Section III introduces in detail a two-stage method for solving the calibration problem that uses a line laser module. A closed-form solution and a nonlinear optimization based on the geometrical constraints are presented. Section IV summarizes the simulation results and actual data. Section V draws conclusions.

\section{PRoblem Definition}

Fig. 1 illustrates the overall configuration of an eye-to-hand system, in which a line laser is attached to the end-effector. The robot base coordinate system is defined as a world coordinate system as follows.

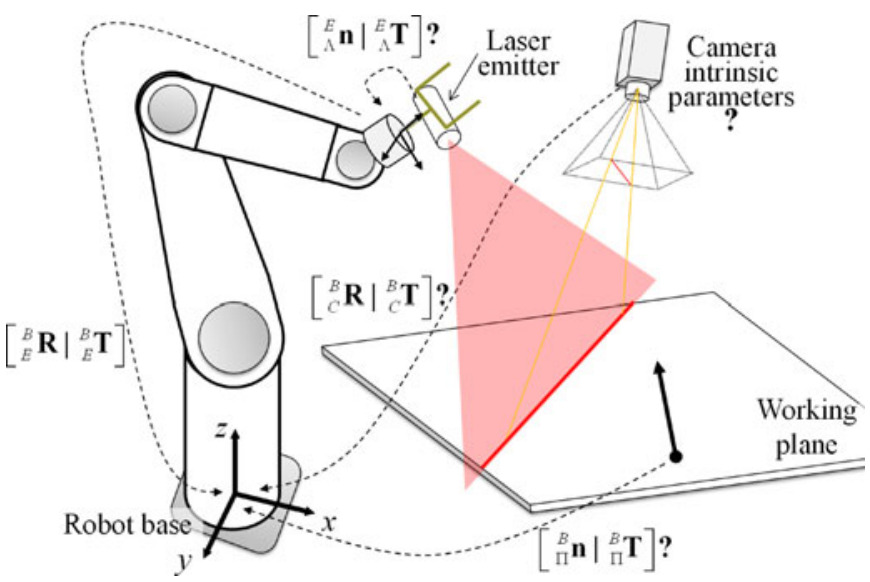

Fig. 1. Overview of an eye-to-hand system with a line laser module.

\section{A. Intrinsic Parameters of Camera}

The camera model describes the relationship between a 3-D position and its projection onto the image plane. A position in the Cartesian coordinate system is denoted ${ }^{C} \mathbf{p}=\left[{ }^{C} x,{ }^{C} y,{ }^{C} z\right]^{T}$ and the direction of the ray from the center of the camera to ${ }^{C} \mathbf{p}$

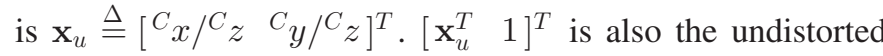
position of the point that the ray intersects with the unit $z$ plane. Based on Brown's distortion model [32], the transformation from the distorted position, $\mathbf{x}_{d}=\left[\begin{array}{ll}x_{d} & y_{d}\end{array}\right]^{T}$, is given by

$\mathbf{x}_{u}=\mathbf{x}_{d}+\left(\kappa_{1} r^{2}+\kappa_{2} r^{4}\right) \mathbf{x}_{d}+\left[\begin{array}{c}2 \rho_{1} x_{d} y_{d}+\rho_{2}\left(r^{2}+2 x_{d}^{2}\right) \\ \rho_{1}\left(r^{2}+2 y_{d}^{2}\right)+2 \rho_{2} x_{d} y_{d}\end{array}\right]$

where $\kappa_{1}$ and $\kappa_{2}$ are two radial distortion parameters; $r=$ $\sqrt{x_{d}^{2}+y_{d}^{2}}$, and $\rho_{1}$ and $\rho_{2}$ are two tangential distortion parameters. When distortion is neglected, all distortion parameters are zero and $\mathbf{x}_{u}=\mathbf{x}_{d}$. The camera pinhole model is a transformation between the image coordinate system and the 3-D coordinate system. The transformation between the distorted ray direction $\mathbf{x}_{d}$ and the observed image position $\mathbf{x}_{n}=[u, v]^{T}$ is

$$
\left[\begin{array}{c}
\mathbf{x}_{d} \\
1
\end{array}\right]=\mathbf{K}^{-1} \cdot\left[\begin{array}{c}
\mathbf{x}_{n} \\
1
\end{array}\right] \text { with } \mathbf{K}=\left[\begin{array}{ccc}
f_{u} & \alpha_{c} \cdot f_{u} & u_{0} \\
0 & f_{v} & v_{0} \\
0 & 0 & 1
\end{array}\right]
$$

where $\mathbf{K}$, the intrinsic matrix, includes five intrinsic parameters, of which $f_{u}$ and $f_{v}$ are the focal lengths in pixels; the aspect ratio is $f_{u} / f_{v} ; \alpha_{c}$ is the screw coefficient; and $\left[\begin{array}{ll}u_{0} & v_{0}\end{array}\right]^{T}$ denotes the principal point, which is assumed to be the center of distortion. This camera model is slightly modified from that introduced by Willson [33]. Since the effective focal length and the centerto-center distance between sensor elements can be combined as a pixel unit focal length for simplicity, this camera model combines their first and fourth transformations in [33] without loss of generality, and the skew coefficient and more distortion parameters are considered.

\section{B. Eye-to-Hand Transformation}

The rigid body transformation involves six degrees of freedom, and so has three translational parameters and three 


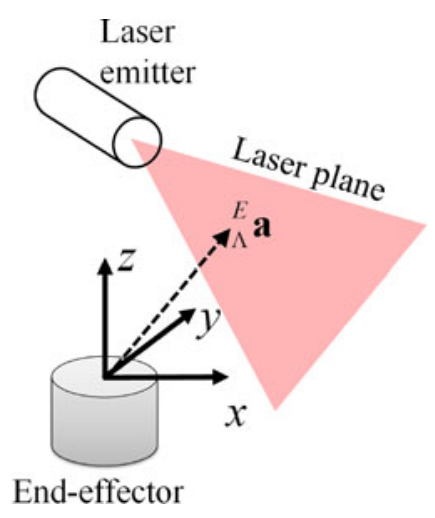

Fig. 2. Laser plane with respect to the end-effector.

rotational parameters. A point ${ }^{C} \mathbf{p}$ in the 3-D Cartesian coordinate system in the camera frame is transformed into the robot base coordinate system by applying

$$
{ }^{B} \mathbf{p}={ }_{C}^{B} \mathbf{R} \cdot{ }^{C} \mathbf{p}+{ }_{C}^{B} \mathbf{T} \text {. }
$$

The rotation matrix is derived from the direction cosine matrix, $\mathbf{R}(\theta, \phi, \varphi)$. The rotation sequence is $\mathrm{Z}-\mathrm{Y}-\mathrm{X}$, where $\theta$ is the rotation about the $z$-axis; $\phi$ denotes rotation about the $y$-axis; and $\varphi$ denotes rotation about the $x$-axis rotation. Transformation from the end-effector frame to the robot base frame is conducted using a rotation matrix ${ }_{E}^{B} \mathbf{R}$ and a translation vector ${ }_{E}^{B} \mathbf{T}$. A robot command determines the hand pose $\left.\left.{ }_{E}^{B} \mathbf{R}\right|_{E} ^{B} \mathbf{T}\right]$ according to forward kinematics. In the following, the robot base coordinate system is defined as the world coordinate system.

\section{Working Plane}

A planar object is defined by a normal vector ${ }_{\Pi}^{B} \mathbf{n}$ and a position ${ }_{\Pi}^{B} \mathbf{T}$ on the plane in the robot base coordinate system. A plane in space has three degrees of freedom and can be generally defined using the following vector:

$$
{ }_{\Pi}^{B} \mathbf{a}=\left({ }_{\Pi}^{B} \mathbf{n}^{T} \cdot{ }_{\Pi}^{B} \mathbf{T}\right)_{\Pi}^{B} \mathbf{n} .
$$

This vector also specifies a position on the plane. Any point ${ }^{B} \mathbf{X}$ on the plane must satisfy the constraint

$$
{ }_{\Pi}^{B} \mathbf{a}^{T}\left({ }^{B} \mathbf{x}-{ }_{\Pi}^{B} \mathbf{a}\right)=0 .
$$

\section{Laser Plane}

Fig. 2 shows the geometrical relationship between the line laser module and the end-effector. In the end-effector coordinate system, the laser plane is specified by a vector ${ }_{\Lambda}^{E} \mathbf{a}=\left({ }_{\Lambda}^{E} \mathbf{n}^{T}\right.$. $\left.{ }_{\Lambda}^{E} \mathbf{T}\right)_{\Lambda}^{E} \mathbf{n}$, which is perpendicular to the laser plane and ends on the laser plane. This laser vector is an extra calibration target in the proposed method. Since the laser module is rigidly installed, the parameters do not change under normal operations.

\section{PRoposed Method}

This section describes in detail the method for calibrating an eye-to-hand system. Calibration is preformed to reduce systematic errors by finding and correcting the parameters that were introduced in Section II.
The line of intersection of two planes in 3-D space is described by its direction and any position on the line. Any point $\mathbf{x}$ on the line of intersection of plane $a$ and plane $b$ satisfying

$$
{ }_{a} \mathbf{a}^{T}\left(\mathbf{x}-{ }_{a} \mathbf{a}\right)=0 \text { and }{ }_{b} \mathbf{a}^{T}\left(\mathbf{x}-{ }_{b} \mathbf{a}\right)=0
$$

and can be expressed as

$$
\mathbf{x}=d \mathbf{u}+\mathbf{x}_{0}
$$

where the direction of the line, $\mathbf{u}$, is given by $\mathbf{u}=\left({ }_{a} \mathbf{a} \times\right.$ $\left.{ }_{b} \mathbf{a}\right) /\left\|_{a} \mathbf{a} \times{ }_{b} \mathbf{a}\right\|$; without loss of generality, let $\mathbf{x}_{0}$ be the point on the line that is closest to the origin of the world coordinate system, such that $\mathbf{u}^{T} \mathbf{x}_{0}=0$. The term $d$ represents the distance from $\mathbf{x}_{0}$ to $\mathbf{x}$.

A laser line stripe is formed by projecting the laser onto the working plane and then captured by the camera. The line strip lies on the three planes (laser plane, working plane, and camerato-stripe plane). The line can be specified as the intersection of any two of the three planes, indicating that this system configuration contains redundant information. Differences between specifications of a single line derived using different planes are caused by systematic errors and/or noise in the measurements. Restated, the systematic parameters can be estimated by tuning them to reduce the differences between the lines that are computed from different intersections. Additionally, forming a batch of projected points enables the effects of noise to be reduced by various optimization methods.

The optimization problem, as described in the next section, is nonlinear. A good initial guess is required to avoid a local minimum and accelerate convergence. A closed-form solution is derived in Section III-A, by exploring the unique hand movement. Since the parameters need not be guessed in advance, the proposed method can be implemented automatically.

\section{A. Closed-Form Solution}

Manipulating the end-effector without rotation can generate parallel laser planes and parallel laser line stripes, which are projected on the working plane. Two nonparallel line stripes on the same working plane virtually cross each other in 3-D space and in the image plane. Two sets of parallel line stripes generate a set of virtual crossing points, which are distributed as a gridlike pattern. The closed-form solution is derived based on these geometrical relationships.

The laser line stripes on the working plane under a single plane pose can only provide 2-D information in 3-D space. Hence, the working plane pose must be changed once or more times to generate abundant 3-D information for calibrating the intrinsic parameters of the camera. If working plane pose cannot be changed, then another plane with a different poses can be put on it.

Fig. 3 shows the relationships among projected laser stripes and their virtual crossing points. For the $p$ th working plane pose, the end-effector maintains the ( $p, m 1)$ th orientation ${ }_{E} \mathbf{R}_{p, m 1}$ and is translated from the position ${ }_{E} \mathbf{T}_{p, m 1}$ to several positions in the $\left(p, m 1, n 1\right.$ )th direction (or vector $\mathbf{k}_{p, m 1, n 1}$ ). These translations generate parallel line stripes. This set of parallel line strips virtually crosses to another set of parallel line stripes, which 


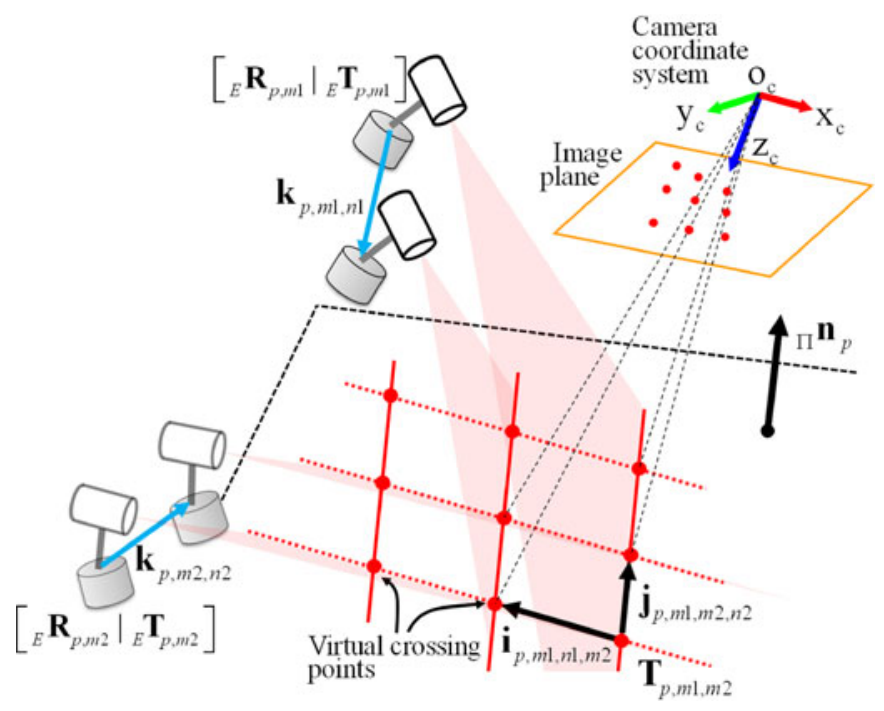

Fig. 3. Projected laser stripes and virtual crossing points.

are generated by moving the end-effector from ${ }_{E} \mathbf{T}_{p, m 2}$ in the $(p, m 2, n 2)$ th direction (or vector $\mathbf{k}_{p, m 2, n 2}$ ) at hand orientation ${ }_{E} \mathbf{R}_{p, m 2}$. The working plane, laser plane at the $(p, m 1)$ th hand pose, and laser plane at $(p, m 2)$ th hand pose all intersect at $\mathbf{T}_{p, m 1, m 2}$. The translation $\mathbf{k}_{p, m 1, n 1}$ moves the crossing point to $\mathbf{T}_{p, m 1, m 2}+\mathbf{i}_{p, m 1, n 1, m 2}$ and the translation $\mathbf{k}_{p, m 1, n 1}$ moves the crossing point to $\mathbf{T}_{p, m 1, m 2}+\mathbf{j}_{p, m 1, m 2, n 2}$. The $(p, m 1, m 2, k)$ th crossing point that is projected onto camera is at image position $\mathbf{x}_{n}$ and its homogeneous coordinate is

$$
\begin{aligned}
\mathbf{x}_{p, m 1, m 2, k}= & {\left[\begin{array}{c}
z \cdot \mathbf{x}_{n} \\
z
\end{array}\right]_{p, m 1, m 2, k}=\mathbf{H}_{p, m 1, m 2} \mathbf{w}_{p, m 1, m 2, k} } \\
= & \mathbf{K}\left[\begin{array}{ccc}
{ }^{C} \mathbf{i}_{p, m 1, n 1, m 2} & { } \mathbf{j}_{p, m 1, m 2, n 2} & { }^{C} \mathbf{T}_{p, m 1, m 2}
\end{array}\right] \\
& \times\left[\begin{array}{c}
w^{i} \\
w^{j} \\
1
\end{array}\right]_{p, m 1, m 2, k}
\end{aligned}
$$

where $w^{i}$ and $w^{j}$ denote scales of a combination according to end-effector translating distance, $z$ is a related scalar, and $\mathbf{H}_{p, m 1, m 2}$ is a homogeneous matrix. Supposing that the endeffector is translated in $N$ directions at each hand orientation, $\mathbf{H}_{p, m 1, m 2}$ then becomes a $3 \times(2 N+1)$ matrix, which includes $N$ vectors $\mathbf{i}, N$ vectors $\mathbf{j}$, and one starting position $\mathbf{T}$.

The closed-form solutions are derived from the measurements of the camera to the movements of the robot. Pure hand translations can eliminate the position terms of parameters in equations. The relationships can then be derived as several linear forms to find angle related terms of parameters. After all directions and orientations have been obtained, the position terms and the scaling factors can be obtained. The step-by-step calculations to obtain the closed-form solution are as follows.

1) Finding Homography Matrixes With Unknown Scales: The entries of a homography matrix can be obtained by eliminating $w$ according to the direct linear transformation method [21], [22]. Let $\overline{\mathbf{h}}^{a}$ be the $a$ th row of $\mathbf{H}$. Equation (8) can be rewritten

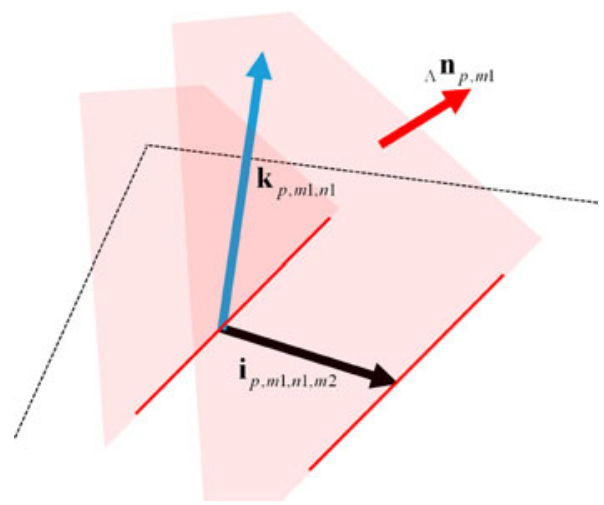

Fig. 4. Projection of an end-effector translating vector along a laser plane on a working plane.

as

$$
\mathbf{Q}_{\mathbf{H}} \cdot \mathbf{x}_{\mathbf{H}} \triangleq\left[\begin{array}{rrr}
\mathbf{w}_{k}^{T} & \mathbf{0} & -u_{k} \mathbf{w}_{k}^{T} \\
\mathbf{0} & \mathbf{w}_{k}^{T} & -v_{k} \mathbf{w}_{k}^{T}
\end{array}\right]_{p, m 1, m 2}\left[\begin{array}{c}
\overline{\mathbf{h}}^{1 T} \\
\overline{\mathbf{h}}^{2} \\
\overline{\mathbf{h}}^{3}{ }^{T}
\end{array}\right]_{p, m 1, m 2}=\mathbf{0} .
$$

Given $K$ points, $\mathbf{Q}_{\mathbf{H}}$ becomes a $2 K \times(6 N+3)$ matrix. The solution $\mathbf{x}_{\mathbf{H}}$ lies in the null space of the matrix $\mathbf{Q}_{\mathbf{H}}$ and can be determined by calculating the eigenvector that corresponds to the smallest eigenvalue of matrix $\mathbf{Q}_{\mathbf{H}}{ }^{T} \mathbf{Q}_{\mathbf{H}}$. The state-ofthe-art eigendecomposition approach is based on singular value decomposition. The estimated matrix $\hat{\mathbf{H}}_{p, m}$ with an unknown scaling factor is equivalent to the homography:

$$
\begin{aligned}
& \mathbf{H}_{p, m 1, m 2}=\lambda_{p, m 1, m 2} \hat{\mathbf{H}}_{p, m 1, m 2} \\
& \triangleq \lambda_{p, m 1, m 2}\left[\begin{array}{llll}
\cdots & C_{\hat{\mathbf{i}}_{p, m 1, n 1, m 2}} & \ldots \\
C_{\hat{\mathbf{j}}_{p, m 1, m 2, n 2}} & \ldots & { }^{C} \hat{\mathbf{T}}_{p, m 1, m 2}
\end{array}\right]
\end{aligned}
$$

For a given working plane pose, all crossing points should be on this working plane even under various hand orientations. Thus, the ratios between values of $\lambda_{p, m 1, m 2}, m 1=1-M, m 2$ : hand orientations have crossing points to $m 1$, can be obtained. Then, only one scaling factor is undetermined for one working plane pose. The $P$ unknown scaling factors can be obtained by the following steps.

2) Finding the Normal Vector of the Laser Plane Relative to the Robot: The translations of the end-effector are related to the movements of the laser line stripe on the working plane by projecting the hand translation vectors to the working plane along the laser plane (see Fig. 4). The projection relationship satisfies

$$
\left(\mathbf{i}_{p, m 1, n 1, m 2}-\mathbf{k}_{p, m 1, n 1}\right)^{T} \cdot{ }_{\Lambda} \mathbf{n}_{p, m 1}=0 .
$$

The vectors, $\mathbf{i}_{p, m 1, n 1, m 2}, n 1=1-N$, are all in the same direction since they are on the parallel line stripes that are generated with the $(p, m 2)$ th hand orientation. Therefore, the vectors can be specified as $\mathbf{i}_{p, m 1, n 1, m 2}=d_{p, m 1, n 1, m 2} \cdot \mathbf{i}_{p, m 1, m 2}$, where 
$\mathbf{i}_{p, m 1, m 2}$ is a unit vector. The distance is

$$
d_{p, m 1, n 1, m 2}=\frac{\mathbf{k}_{p, m 1, n 1}^{T} \cdot{ }_{\Lambda} \mathbf{n}_{p, m 1}}{\mathbf{i}_{p, m 1, m 2}^{T} \cdot{ }_{\Lambda} \mathbf{n}_{p, m 1}}
$$

Then, the ratio of the $n 1$ th distances to the 1 th distance is

$$
\frac{d_{p, m 1, n 1, m 2}}{d_{p, m 1,1, m 2}}=\frac{\mathbf{k}_{p, m 1, n 1}^{T} \cdot{ }_{\Lambda} \mathbf{n}_{p, m 1}}{\mathbf{k}_{p, m 1,1}^{T} \cdot{ }_{\Lambda} \mathbf{n}_{p, m 1}}
$$

This ratio can also be determined as

$$
\begin{aligned}
\frac{d_{p, m 1, n 1, m 2}}{d_{p, m 1,1, m 2}}= & \frac{\mathbf{i}_{p, m 1, n 1, m 2}^{T} \cdot \mathbf{i}_{p, m 1,1, m 2}}{\mathbf{i}_{p, m 1,1, m 2}^{T} \cdot \mathbf{i}_{p, m 1,1, m 2}} \\
& =\frac{\hat{\mathbf{i}}_{p, m 1, n 1, m 2}^{T} \cdot \hat{\mathbf{i}}_{p, m 1,1, m 2}}{\hat{\mathbf{i}}_{p, m 1,1, m 2}^{T} \cdot \hat{\mathbf{i}}_{p, m 1,1, m 2}} .
\end{aligned}
$$

Combining (13) and (14) yields a constraint equation:

$$
\begin{array}{r}
\left(\hat{\mathbf{i}}_{p, m 1, n 1, m 2}^{T} \cdot \hat{\mathbf{i}}_{p, m 1,1, m 2} \cdot \mathbf{k}_{p, m 1,1}^{T}-\hat{\mathbf{i}}_{p, m 1, n 1, m 2}^{T} \cdot \hat{\mathbf{i}}_{p, m 1,1, m 2}\right. \\
\left.\cdot \mathbf{k}_{p, m 1, n 1}^{T}\right) \cdot{ }_{\Lambda} \mathbf{n}_{p, m 1}=0 .
\end{array}
$$

For $n 1=2-N, N-1$ equations are formed to determine the laser normal vector at ${ }_{\Lambda} \mathbf{n}_{p, m 1}$. Similarly,

$$
\begin{array}{r}
\left(\hat{\mathbf{j}}_{p, m 1, m 2, n 2}^{T} \cdot \hat{\mathbf{j}}_{p, m 1, m 2,1} \cdot \mathbf{k}_{p, m 2,1}^{T}-\hat{\mathbf{j}}_{p, m 1, m 2,1}^{T} \cdot \hat{\mathbf{j}}_{p, m 1, m 2,1}\right. \\
\left.\cdot \mathbf{k}_{p, m 2, n 2}^{T}\right) \cdot{ }_{\Lambda} \mathbf{n}_{p, m 2}=0
\end{array}
$$

$n 2=2-N$, yields ${ }_{\Lambda} \mathbf{n}_{p, m 2}$.

From all normal vectors of the laser plane in the robot base coordinate system, ${ }_{\Lambda}^{B} \mathbf{n}_{p, m}$, and the corresponding hand orientations, ${ }_{E}^{B} \mathbf{R}_{p, m}, p=1-P, m=1-M$, the laser normal vector ${ }_{\Lambda}^{E} \mathbf{n}$ with respect to the end-effector coordinate system can be obtained by solving the simultaneous equations

$$
\mathbf{A}_{\Lambda} \mathbf{x}_{\Lambda} \triangleq\left[\begin{array}{c}
{ }_{E}^{B} \mathbf{R}_{1,1} \\
\vdots \\
{ }_{E}^{B} \mathbf{R}_{P, M}
\end{array}\right]{ }_{\Lambda}^{E} \mathbf{n}=\mathbf{b}_{\mathbf{u}} \triangleq\left[\begin{array}{c}
{ }_{\Lambda}^{B} \mathbf{n}_{1,1} \\
\vdots \\
{ }_{\Lambda}^{B} \mathbf{n}_{P, M}
\end{array}\right]
$$

using the least-squares method, and normalizing the solution to $\left|{ }_{\Lambda}^{E} \mathbf{n}\right|=1$. According to (17), only one orientation is required to calculate a unique ${ }_{\Lambda}^{E} \mathbf{n}$. However, to find the unique laser beam position ${ }_{\Lambda}^{E} \mathbf{T}$ in the following step, at least two orientations are required.

3) Finding the Intrinsic Matrix and the Hand-to-Eye Rotation Matrix: A line stripe lies on its corresponding laser plane. Hence,

$$
\begin{aligned}
& { }_{\Lambda}^{B} \mathbf{n}_{p, m 2}^{T} \cdot{ }^{B} \mathbf{i}_{p, m 1, n 1, m 2} \\
& =\lambda_{p}{ }_{\Lambda}^{B} \mathbf{n}_{p, m 2}^{T} \cdot{ }_{C}^{B} \mathbf{R} \mathbf{K}^{-1} \cdot \hat{\mathbf{i}}_{p, m 1, n 1, m 2}=0
\end{aligned}
$$

and

$$
\begin{aligned}
& { }_{\Lambda}^{B} \mathbf{n}_{p, m 1}^{T} \cdot{ }^{B} \mathbf{j}_{p, m 1, m 2, n 2} \\
& \quad=\lambda_{p}{ }_{\Lambda}^{B} \mathbf{n}_{p, m 1}^{T} \cdot{ }_{C}^{B} \mathbf{R} \mathbf{K}^{-1} \cdot \hat{\mathbf{j}}_{p, m 1, m 2, n 2}=0 .
\end{aligned}
$$

Let $\hat{\mathbf{G}}=\left(\mathbf{K}_{B}^{C} \mathbf{R}\right)^{-1}$ and denote the $a$ th row of matrix $\hat{\mathbf{G}}$ as $\hat{\overline{\mathbf{g}}}^{a}$. Equations (18) and (19) can be written in another form

$$
\mathrm{Q}_{\mathrm{G}} \mathrm{x}_{\mathrm{G}} \stackrel{\triangleq}{=}
$$

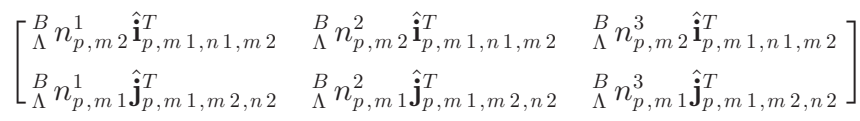$$
\left[\begin{array}{l}
\hat{\mathbf{g}}^{{ }^{T}} \\
\hat{\mathbf{g}}^{2^{T}} \\
\hat{\overline{\mathbf{g}}}^{3^{T}}
\end{array}\right]=\mathbf{0}
$$

where ${ }_{\Lambda}^{B} n_{p, m}^{a}$ denotes the $a$ th entries of laser normal vector ${ }_{\Lambda}^{B} \mathbf{n}_{p, m}$. Let $\hat{M}$ be the number of that two hand orientations have crossing relationship in each working plane pose. For all $P$ plane poses and $N$ translations under each hand orientation, $\mathbf{Q}_{\mathrm{G}}$ becomes a $(2 P \hat{M} N) \times 9$ matrix. Solution $x_{G}$ lies in the null space of the matrix $\mathrm{Q}_{\mathrm{G}}$. The solution can be derived by calculating the eigenvector that corresponds to the smallest eigenvalue of matrix $\mathbf{Q}_{\mathbf{G}}{ }^{T} \mathbf{Q}_{\mathbf{G}}$. Since each row of the rotation matrix ${ }_{B}^{C} \mathbf{R}$ is a unit vector, and the last entry of $\mathbf{K}$ is 1 , the solution is scaled to satisfy $g_{31}^{2}+g_{32}^{2}+g_{33}^{2}=1$. RQ-decomposition is applied to separate the homogeneous matrix $\mathbf{G}$ into the intrinsic matrix $\mathbf{K}$ and the rotation matrix ${ }_{B}^{C} \mathbf{R}$.

4) Finding the Scaling Factors and the Laser Position and the Camera Position Relative to the Robot: The $p$ th working plane, the laser plane associated with the $(p, m 1, k 1)$ th hand pose, and the laser plane associated with the $(p, m 2, k 2)$ th hand pose intersect at the $(p, m 1, m 2, k)$ th virtual crossing point

$$
{ }_{\Pi}^{C} \mathbf{p}_{p, m 1, m 2, k}=\lambda_{p} \mathbf{K}^{-1} \hat{\mathbf{H}}_{p, m 1, m 2} \mathbf{w}_{p, m 1, m 2, k} .
$$

Hence,

${ }_{\Lambda}^{C} \mathbf{n}_{p, m 1}^{T}\left({ }_{\Pi}^{C} \mathbf{p}_{p, m 1, m 2, k}-{ }_{B}^{C} \mathbf{R}\left({ }_{E}^{B} \mathbf{R}_{p, m 1}{ }_{\Lambda}^{E} \mathbf{a}-{ }_{E}^{B} \mathbf{T}_{p, m 1, k 1}\right)-{ }_{B}^{C} \mathbf{T}\right)$
$=0 \quad(22)$

and

${ }_{\Lambda}^{C} \mathbf{n}_{p, m 2}^{T}\left({ }_{\Pi}^{C} \mathbf{p}_{p, m 1, m 2, k}-{ }_{B}^{C} \mathbf{R}\left({ }_{E}^{B} \mathbf{R}_{p, m 22}{ }_{\Lambda}^{E} \mathbf{a}-{ }_{E}^{B} \mathbf{T}_{p, m 2, k 2}\right)-{ }_{B}^{C} \mathbf{T}\right)$

$$
=0 \text {. }
$$

Consequently,

$$
\begin{gathered}
{\left[\begin{array}{lll}
{ }_{\Lambda}^{C} \mathbf{n}_{p, m 1}^{T} & 1 & -{ }_{\Lambda}^{C} \mathbf{n}_{p, m 1}^{T} \mathbf{K}^{-1} \hat{\mathbf{H}}_{p, m 1, m 2} \mathbf{w}_{p, m 1, m 2, k} \\
{ }_{\Lambda}^{C} \mathbf{n}_{p, m 2}^{T} & 1 & -{ }_{\Lambda}^{C} \mathbf{n}_{p, m 2}^{T} \mathbf{K}^{-1} \hat{\mathbf{H}}_{p, m 1, m 2} \mathbf{w}_{p, m 1, m 2, k}
\end{array}\right]\left[\begin{array}{c}
{ }_{B}^{C} \mathbf{T} \\
s \\
\lambda_{p}
\end{array}\right]} \\
=\left[\begin{array}{ll}
{ }_{\Lambda}^{C} \mathbf{n}_{p, m 1}^{T}{ }_{B}^{C} \mathbf{R}_{E}^{B} \mathbf{T}_{p, m 1, k 1} \\
{ }_{\Lambda}^{C} \mathbf{n}_{p, m 2}^{T} & { }_{B}^{C} \mathbf{R}_{E}^{B} \mathbf{T}_{p, m 2, k 2}
\end{array}\right]
\end{gathered}
$$

where the scalar $s$ satisfies ${ }_{\Lambda}^{E} \mathbf{a}=s \cdot{ }_{\Lambda}^{E} \mathbf{n}$. The $P \times \hat{M} \times K$ sets of data yield the equation

$$
\mathbf{A}_{\mathbf{T}, \lambda} \mathbf{x}_{\mathbf{T}, \lambda}=\mathbf{b}_{\mathbf{T}, \lambda}
$$

where $\mathbf{A}_{\mathbf{T}, \lambda}$ is a matrix of size of $(2 P \hat{M} K) \times(4+P), \mathbf{b}_{\mathbf{T}, \lambda}$ denotes a vector of size of $2 P \hat{M} K$, and $\mathbf{x}_{\mathbf{T}, \lambda}={ }_{C}^{B} \mathbf{T}^{T} s$ $\left.\lambda_{1} \cdots \lambda_{P}\right]^{T}$. This equation can be solved using the least 

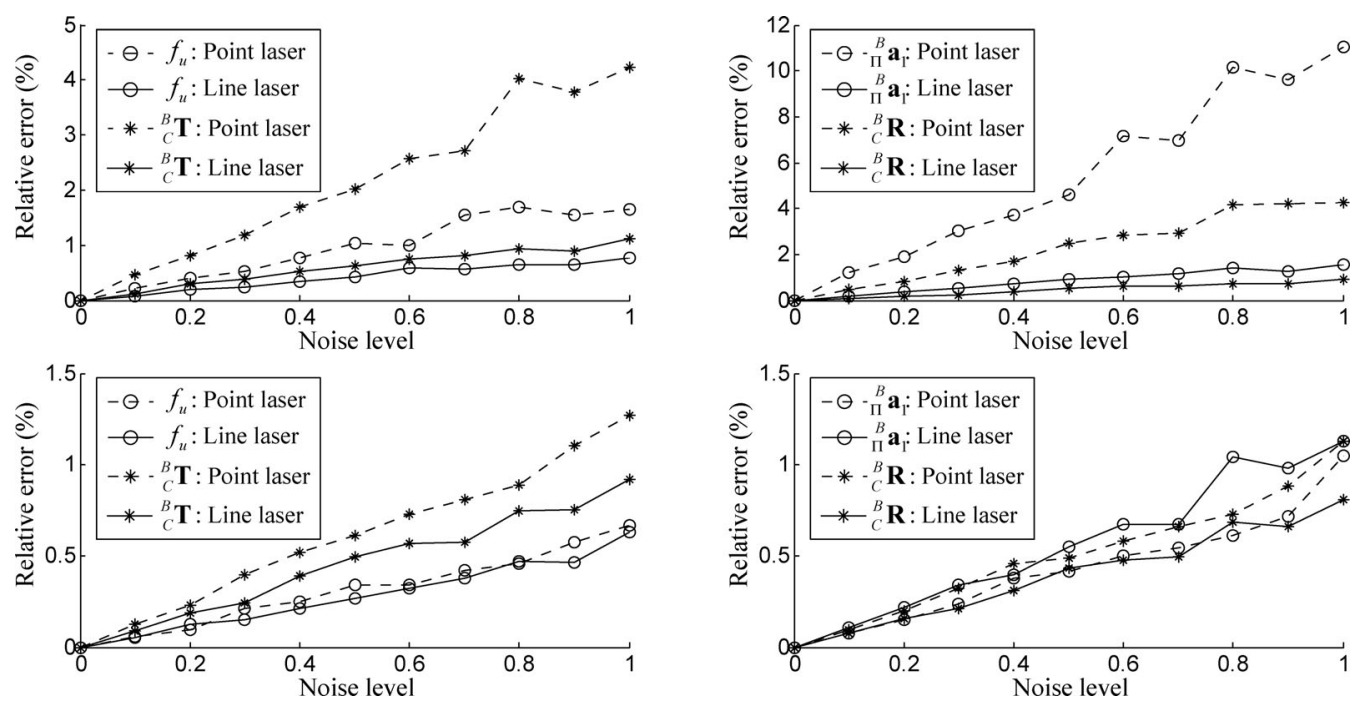

Fig. 5. Relative errors with respect to the noise level. (Left) Focal length and camera position errors. (Right) Working plane pose and camera rotation errors. (Top) Closed-form solution. (Bottom) Nonlinear optimization.

squares method. The laser position in the end-effector frame and the camera position in the robot base frame are then obtained. The homography matrices related to robot dimension are scaled as $\mathbf{H}_{p, m}=\lambda_{p} \hat{\mathbf{H}}_{p, m}$.

5) Finding the Working Planes: Vectors ${ }^{C} \mathbf{i}_{p, m 1, n 1, m 2}=$ $\lambda_{p} \mathbf{K}^{-1} \cdot \hat{\mathbf{i}}_{p, m 1, n 1, m 2}$ lie on the $p$ th plane and so do vectors ${ }^{C} \mathbf{j}_{p, m 1, m 2, n 2}=\lambda_{p} \mathbf{K}^{-1} \cdot \hat{\mathbf{j}}_{p, m 1, m 2, n 2}$. The normal vector of the working plane, ${ }_{\Pi}^{C} \mathbf{n}_{p}$, is orthogonal to these vectors. The virtual crossing point, ${ }^{C} \mathbf{T}_{p, m 1, m 2}=\lambda_{p} \mathbf{K}^{-1} \cdot \hat{\mathbf{T}}_{p, m 1, m 2}$, is on the $p$ th plane and the position of the plane can be determined from the position of one of these crossing points or by averaging them.

\section{B. Nonlinear Optimization}

The above closed-form solution provides a good estimate of parameters of the system. However, the error can propagate throughout the procedure. A nonlinear optimization problem is introduced to refine the estimation. The projected laser line is constrained within the image plane under various arm poses, yielding the following optimization problem:

$$
\begin{aligned}
\min _{S} \sum_{p=1}^{P} \sum_{m=1}^{M} \sum_{k=1}^{K} \sum_{l=1}^{L} \operatorname{dist} & \left(\mathbf{x}_{u}\left(\left[\begin{array}{l}
u \\
v
\end{array}\right]_{p, m, k, l}\right),\right. \\
& \left.\left.\operatorname{line}\left(S,\left.{ }_{E}^{B} \mathbf{R}_{p, m}\right|_{E} ^{B} \mathbf{T}_{p, m, k}\right]\right)\right)
\end{aligned}
$$

where $S$ is the set of parameters to be refined, which includes $f_{u}, f_{v}, \alpha_{c}, u_{0}, v_{0}, \kappa_{1}, \kappa_{2}, \rho_{1}, \rho_{2},{ }_{B}^{C} \theta,{ }_{B}^{C} \phi,{ }_{B}^{C} \varphi,{ }_{B}^{C} \mathbf{T},{ }_{\Lambda}^{E} \mathbf{a}$, and ${ }_{\Pi}^{B} \mathbf{a}_{p}, p=1, \ldots, P ;\left[\begin{array}{ll}u & v\end{array}\right]_{p, m, k, l}^{T}$ is an image position on the observed laser stripe in the $(p, m, k)$ th image; $\mathbf{x}_{u}(\cdot)$ denotes the inverse of the projection from the image position to the unit $z$ plane in the camera frame, given by (2) and (1); line $(\cdot)$ denote the projection of a line onto the unit $z$ plane in the camera frame, and this line is the laser line that is calculated using the robot command $\left.\left.{ }_{E}^{B} \mathbf{R}_{p, m}\right|_{E} ^{B} \mathbf{T}_{p, m, k}\right]$; and parameter set $S$, and $\operatorname{dist}(a, b)$ denotes the distance from a point $a$ to a line $b$.
Equation (26) can be solved by the Levenberg-Marquardt method. An initial guess for $S$ is required and can be made using the previously described closed-form solution. Second, if all of the system parameters can be obtained from specifications or design settings, these values must be close to the real values and can be used as an initial guess in the optimization.

\section{EXPERIMENTAL RESULTS}

\section{A. Simulations}

The proposed method is validated under various noise levels and various numbers of samples. It is compared to the method that uses a single-point laser. The relationship between the camera and the robot is given by ${ }_{C}^{B} \theta=-90^{\circ},{ }_{C}^{B} \phi=-10^{\circ}$, ${ }_{C}^{B} \varphi=-170^{\circ}$, and ${ }_{C}^{B} T=[510,151,430]^{T}(\mathrm{~mm})$. The line laser is at ${ }_{\Lambda}^{E} a=[8.73,17.48,43.65]^{T}(\mathrm{~mm})$ relative to the end-effector. The three working plane poses are ${ }_{\Pi}^{B} \mathbf{a}_{1}=[48.68$, $31.47,-153.81]^{T}(\mathrm{~mm}),{ }_{\Pi}^{B} \mathbf{a}_{2}=[152.41,138.34,-163.92]^{T}$ $(\mathrm{mm})$, and ${ }_{\Pi}^{B} \mathbf{a}_{3}=[-4.0,8.40,29.80]^{T}(\mathrm{~mm})$. The intrinsic parameters of the camera are chosen as $f_{u}=1000, f_{v}=1000$, $\alpha_{c}=0, u_{0}=320$, and $v_{0}=240$. The single-point laser is at $[0,-19.90,60.14]^{T}(\mathrm{~mm})$ and pointing in direction $[0.98,0.02$, $-0.20]^{T}$, which is on the laser plane ${ }_{\Lambda}^{E} \mathbf{a}$.

In the following tests, the relative errors of one focal length are calculated as $\left|f_{u, \text { estimated }}-f_{u \text {,true }}\right| / f_{u, \text { true }}$ (percentage). The hand-eye rotation and translation errors are the Frobenius norms of the difference between the true and estimated ${ }_{C}^{B} \mathbf{T}$ and ${ }_{C}^{B} \mathbf{R}$. Additionally, the relative percentage errors are calculated as $\left\|\mathbf{T}_{\text {estimated }}-\mathbf{T}_{\text {true }}\right\|_{F} /\left\|\mathbf{T}_{\text {true }}\right\|_{F}$ and $\| \mathbf{R}_{\text {estimated }}-$ $\mathbf{R}_{\text {true }}\left\|_{F} /\right\| \mathbf{R}_{\text {true }} \|_{F}$. The relative percentage errors of a plane pose are calculated as $\left\|_{\Pi}^{B} \mathbf{a}_{\text {estimated }}-{ }_{\Pi}^{B} \mathbf{a}_{\text {true }}\right\|_{F} /\left\|_{\Pi}^{B} \mathbf{a}_{\text {true }}\right\|_{F}$. For each condition, 100 independent trials are performed and the average of the errors is taken.

The first test is conducted to evaluate the performances under perturbations of different noise levels. Three types of noise are added at a particular time in each trial. They are image position error (0-1 pixel), hand position error $(0-1 \mathrm{~mm})$, and hand orientation error $\left(0-0.05^{\circ}\right)$. Fig. 5 summarizes the performance of 


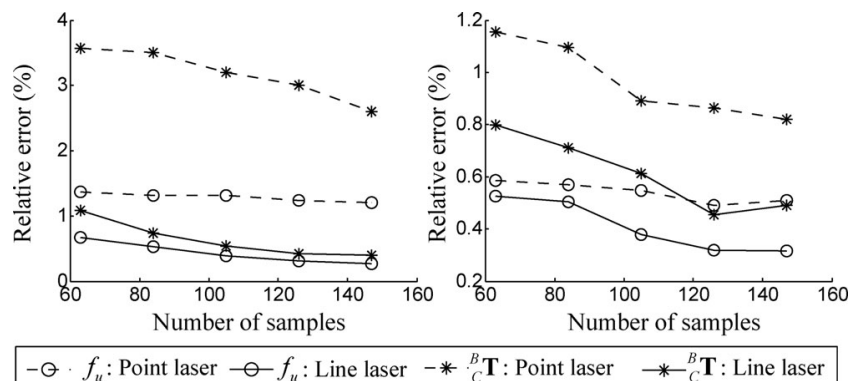

Fig. 6. Relative errors with respect to the number of samples. (Left) Closedform solution and (right) nonlinear optimization.

the closed-form solution and optimization. For each of the three working plane poses, three hand orientations, each associated with ten pure translating points, yielding a total of 90 poses, are generated independently for each trial. The results verify that the proposed method can be adopted to calibrate simultaneously hand-eye-workspace geometrical relationships and the intrinsic parameters of the camera. Furthermore, the accuracy is increased after the optimization procedure. The results reveal that the proposed method that uses a line laser outperforms that uses a point laser for values of all parameters, except the working plane poses of the nonlinear optimization solution.

The number of data also influences the accuracy of the proposed method. The performance of the proposed method with different numbers of samples is compared to that of the method using a single-point laser. Zero mean Gaussian noise with standard deviations of 1 pixel, $1 \mathrm{~mm}$, and $0.05^{\circ}$ is added to each image position of point, each position of the hand, and each orientation of the hand, respectively. Different numbers of hand orientations produce different numbers of samples with measurements of projections in images. Twenty-one to forty-nine hand poses for each of the three working plane poses are generated; therefore, the number of samples varies from 63 to 147. For each number of samples, 100 independent trials are performed and the average of the relative errors is determined. Fig. 6 summarizes the results for one focal length, $f_{u}$, and the camera-to-robot position, ${ }_{C}^{B} \mathbf{T}$. The errors decrease as the number of samples increases. Since information of data is limited, the error of using single-point laser is larger than that generated using the line laser. Notably, a comparison between the results of the closed-form solution obtained using a line laser and those obtained using a point laser shows that the former yields an error in the focal length that is about one third and an error in the camera position that is about one-fifth those generated by the latter. After optimization, these error ratios become $3 / 4$ and $3 / 5$.

\section{B. Real Data}

Fig. 7 shows the experimental setup. The manipulator is a TX60 with a controller, a CS8, that is manufactured by Stäubli Inc. The camera is a Panasonic WV-CP 480 charge-coupled device camera with an adjustable lens. The camera signal in NTSC format is transmitted to the frame grabber, RTV-24, via a coaxial cable. The frame grabber card is embedded in the

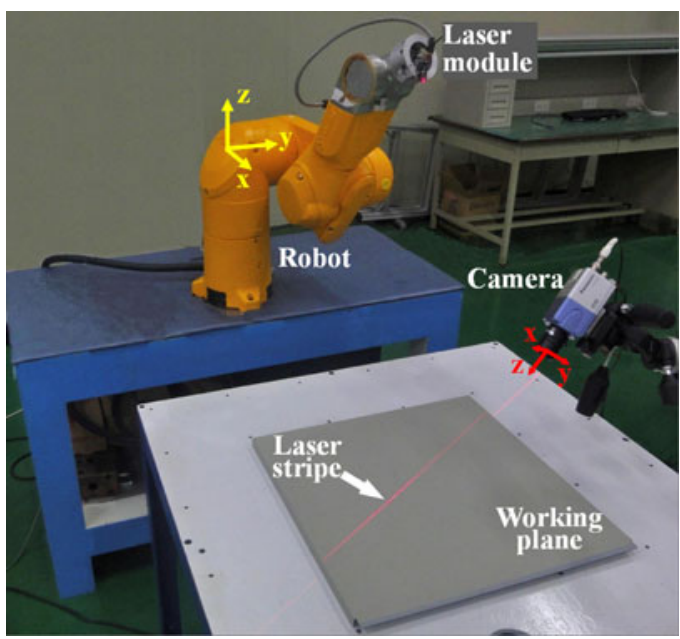

Fig. 7. Overview of the experimental setup.

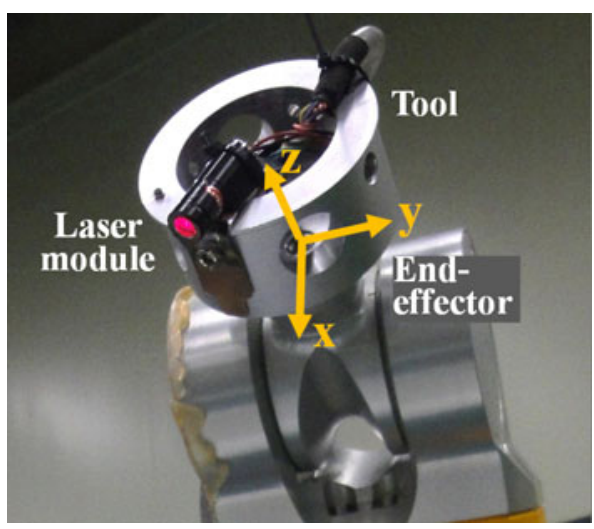

Fig. 8. Line laser module attached to the end-effector.

computer. A Visual C program in the computer can save images, send robot commands to the CS8 controller, and receive the status of the robot from the controller over an Ethernet. A planar plate, of dimensions $50 \times 50 \mathrm{~cm}$, is placed roughly $100 \mathrm{~cm}$ away from the manipulator. In this configuration, the camera cannot see any part of the manipulator. The line laser module is a laser diode with an adjustable lens and is attached on the robot tool (see Fig. 8).

The working plane is orientated at three poses. For each plane pose, the end-effector has three orientations and in each orientation, it starts from one particular position and moves sequentially in three directions. The end-effector stops at three positions in each direction. A total of three working plane poses and 70 hand poses per plane were generated. All movements were designed to project the laser onto the working plane, and corresponding images were saved.

Two tests were performed in this experiment using different numbers of samples. Data were applied to the proposed closedform solutions to obtain initial values of parameters of the system. Next, the proposed nonlinear optimization method was used to refine these values. Table I summarizes the results. Test 1 uses data of three planes and 50 samples per plane. The first column of Table I in Test 1 presents estimates of the closed-form 
TABLE I

Results With Real Data of Different Number of SAMPLES Using a LiNE LASER

\begin{tabular}{|c|c|c|c|c|c|c|}
\hline \multirow{2}{*}{ Parameter } & \multicolumn{3}{|c|}{$\begin{array}{c}\text { Test } 1: 150 \text { samples } \\
\text { (3 plane poses, } 50 \text { samples per plane pose) }\end{array}$} & \multicolumn{2}{|c|}{$\begin{array}{c}\text { Test } 2: 210 \text { samples } \\
\text { (3 plane poses, } 70 \text { samples per plane pose) }\end{array}$} & \multirow{2}{*}{ Unit } \\
\hline & Closed-form solution & Optimization & $\sigma$ & Optimization & $\sigma$ & \\
\hline$\left(f_{u}, f_{v}\right)$ & $(1454.30,1296.65)$ & $(1419.45,1413.01)$ & $(16.26,12.56)$ & $(1277.02,1284.02)$ & $(7.38,5.84)$ & pixel \\
\hline$\alpha_{\mathrm{c}}$ & -0.1180 & -0.0638 & 0.0038 & -0.0287 & 0.0020 & 1 \\
\hline$\left(u_{0}, v_{0}\right)$ & $(231.12,88.97)$ & $(224.67,132.02)$ & $(7.25,5.59)$ & $(228.27,198.04)$ & $(6.46,6.21)$ & pixel \\
\hline$\left(k_{1}, k_{2}\right)$ & $(0,0)$ & $(0.7384,-0.1729)$ & $(0.0894,0.9454)$ & $(0.5104,0.2931)$ & $(0.0538,0.8900)$ & 1 \\
\hline$\left(p_{1}, p_{2}\right)$ & $(0,0)$ & $(-0.0835,-0.0741)$ & $(0.0139,0.0129)$ & $(-0.0352,-0.0431)$ & $(0.0114,0.0120)$ & 1 \\
\hline$\left({ }_{C}^{B} \theta,{ }_{C}^{B} \phi,{ }_{C}^{B} \varphi\right)$ & $(144.85,-24.24,-149.13)$ & $(151.13,-21.34,-152.33)$ & $(0.38,0.59,0.78)$ & $(152.73,-21.41,-152.88)$ & $(0.25,0.50,0.47)$ & deg. \\
\hline${ }_{C}^{B} \mathbf{T}^{T}$ & {$[812.94,137.62,7.54]$} & {$[829.95,142.43,23.23]$} & {$[3.97,4.02,11.88]$} & {$[841.62,136.90,-8.68]$} & {$[2.86,2.33,7.87]$} & $\mathrm{mm}$ \\
\hline${ }_{\Lambda}^{E} \mathbf{a}^{T}$ & {$[-3.57,14.21,42.91]$} & {$[-3.77,15.19,46.01]$} & {$[0.09,0.40,1.01]$} & {$[-3.37,13.87,41.75]$} & {$[0.03,0.12,0.34]$} & $\mathrm{mm}$ \\
\hline${ }_{\Pi}^{B} \mathbf{a}_{1}^{T}$ & {$[-1.18,-7.86,-173.04]$} & {$[-1.03,-2.56,-174.02]$} & {$[1.03,0.95,3.40]$} & {$[-0.61,-3.84,-182.44]$} & {$[0.39,0.55,2.01]$} & $\mathrm{mm}$ \\
\hline${ }_{\Pi}^{B} \mathbf{a}_{2}^{T}$ & {$[13.33,-51.16,-235.07]$} & {$[11.07,-43.22,-229.71]$} & {$[2.11,1.43,3.86]$} & {$[10.45,-45.10,-231.95]$} & {$[0.64,0.63,2.11]$} & $\mathrm{mm}$ \\
\hline${ }_{\Pi}^{B} \mathbf{a}_{3}{ }^{T}$ & {$[63.65,37.94,-349.72]$} & {$[46.26,34.99,-319.95]$} & {$[0.57,0.77,3.12]$} & {$[46.81,31.64,-325.02]$} & {$[1.34,0.93,2.46]$} & $\mathrm{mm}$ \\
\hline Cost & 0.6113 & \multicolumn{2}{|c|}{0.0024} & \multicolumn{2}{|c|}{0.0021} & 1 \\
\hline Error & 22.0374 & \multicolumn{2}{|c|}{1.4448} & \multicolumn{2}{|c|}{0.5230} & $\mathrm{~mm}$ \\
\hline
\end{tabular}

TABLE II

Result With Real Data Using a Single-Point LASER

\begin{tabular}{cccc}
\hline \hline \multirow{2}{*}{ Parameter } & \multicolumn{2}{c}{ 210 samples } & \\
& (3 plane poses, 70 samples per plane pose) & Unit \\
\cline { 2 - 3 }$\left(f_{u}, f_{v}\right)$ & $(1146.11,1136.00)$ & $(30.45,27.61)$ & pixel \\
$\alpha_{\mathrm{c}}$ & 0.0015 & 0.0047 & 1 \\
$\left(u_{0}, v_{0}\right)$ & $(279.74,214.38)$ & $(30.05,26.68)$ & pixel \\
$\left(k_{1}, k_{2}\right)$ & $(0.3337,0.3895)$ & $(0.1304,0.7700)$ & 1 \\
$\left(p_{1}, p_{2}\right)$ & $(-0.0088,-0.0073)$ & $(0.0071,0.0062)$ & 1 \\
$\left({ }_{C}^{B} \theta,{ }_{C}^{B} \phi,{ }^{B} \varphi\right)$ & $(153.85,-20.10,-153.43)$ & $(0.47,1.46,1.25]$ & deg. \\
${ }_{C}^{B} \mathbf{T}^{T}$ & {$[846.14,129.73,-32.12]$} & {$[8.38,5.92,17.71]$} & $\mathrm{mm}$ \\
${ }_{\Pi}^{B} \mathbf{a}_{1}{ }^{T}$ & {$[-1.09,-4.07,-179.36]$} & {$[0.52,0.57,2.79]$} & $\mathrm{mm}$ \\
${ }_{\Pi}^{B} \mathbf{a}_{2}{ }^{T}$ & {$[8.63,-45.80,-223.95]$} & {$[0.80,0.86,2.85]$} & $\mathrm{mm}$ \\
${ }_{\Pi}^{B} \mathbf{a}_{3}{ }^{T}$ & {$[44.42,31.20,-319.78]$} & {$[1.57,1.52,2.88]$} & $\mathrm{mm}$ \\
\hline Error & \multicolumn{2}{c}{7.3502} & $\mathrm{~mm}$ \\
\hline \hline
\end{tabular}

solution that is described in Section III-A. The second column presents the estimates of nonlinear optimization in Test 1 . The third column presents the standard deviations of the estimates of the nonlinear optimization, indicating the uncertainty of the refined result. Test 2 presents results of nonlinear optimization of using all data in right-hand columns. The second-last row of the Table I, labeled Cost, presents the average costs introduced in (26). Twenty points on the working plane are observed by the camera and their positions are determined according to the system model and the estimated parameters. To show the positioning accuracy of the results, the distance from each point to the end-effector is compared to the one measured by a vernier caliper, which has $1500-\mathrm{mm}$ maximum measuring range. The average errors between calculated and measured distances are in the last row, labeled Error. The results are consistent with the simulation results, which revealed that the uncertainty (standard deviation) and error decline as the number of data increases. Table II shows the result with 210 samples using a single-point laser for comparison and it proves that using the line laser is more accurate.

\section{CONCLUSION}

A novel calibration method using a line laser module is proposed for accurately estimating the intrinsic parameters of the camera and the geometrical relationships among a camera, a robot, and a working plane. Since the laser is rigidly mounted on the robot and the working plane is fixed at each plane pose, the laser stripes of the camera measurements and those projected on the working plane must satisfy the geometrical constraints. Based on these constraints, the proposed closed-form solution yields a global solution as initial values of parameters, which are then refined by the nonlinear optimization to enhance accuracy. Simulations are performed to validate both stages of the calibration method and analyze its performance under various conditions. Experimental results reveal that the proposed method is effective when a camera cannot see a hand. Furthermore, the results of the simulation and experiment are compared with results obtained using a single-point laser. The comparison indicates that using the line laser is more accurate.

\section{REFERENCES}

[1] G. Flandin, F. Chaumette, and E. Marchand, "Eye-in-hand/eye-to-hand cooperation for visual servoing," in Proc. IEEE Int. Conf. Robot. Autom., vol. 3, San Francisco, CA, Apr. 2000, pp. 2741-2746.

[2] A. Muis and K. Ohnishi, "Eye-to-hand approach on eye-in-hand configuration within real-time visual servoing," IEEE/ASME Trans. Mechatronics, vol. 10, no. 4, pp. 404-410, Aug. 2005.

[3] S. Hutchinson, G. D. Hager, and P. I. Corke, "A tutorial on visual servo control," IEEE Trans. Robot. Autom., vol. 12, no. 5, pp. 651-670, Oct. 1996.

[4] I.-W. Park, B.-J. Lee, S.-H Cho, Y.-D. Hong, and J.-H. Kim, "Laser-based kinematic calibration of robot manipulator using differential kinematics," IEEE/ASME Trans. Mechatronics, vol. 17, no. 6, pp. 1059-1067, Dec. 2012.

[5] J. Heikkila and O. Silven, "A four-step camera calibration procedure with implicit image correction," in Proc. IEEE Conf. Comput. Vision Pattern Recognit., Jun. 1997, pp. 1106-1112. 
[6] Z. Zhang, "A flexible new technique for camera calibration," IEEE Trans. Pattern Anal. Mach. Intell., vol. 22, no. 11, pp. 1330-1334, Nov. 2000.

[7] P. F. Sturm and S. J. Maybank, "On plane-based camera calibration: A general algorithm, singularities, applications," in Proc. IEEE Int. Conf. Comput. Vision Pattern Recognit., Fort Collins, CO, 1999, pp. 432-437.

[8] J.-Y. Bouguet, Camera calibration toolbox for MATLAB, Jul. 2010. Available: http://www.vision.caltech.edu/bouguetj/calib_doc/

[9] Z. Zhang, "Camera calibration with one-dimensional objects," IEEE Trans. Pattern Anal. Mach. Intell., vol. 26, no. 7, pp. 892-899, Jul. 2004.

[10] T. Echigo, "A camera calibration technique using sets of parallel lines," in Proc. Int. Workshop Ind. Appl. Mach. Intell. Vision, Tokyo, Japan, Apr. 1989, pp. 151-156.

[11] Y. C. Shiu and S. Ahmad, "Calibration of wrist-mounted robotic sensors by solving homogeneous transform equations of the form $\mathrm{AX}=\mathrm{XB}$," IEEE Trans. Robot. Autom., vol. 5, no. 1, pp. 16-29, Feb. 1989.

[12] R. Y. Tsai and R. K. Lenz, "A new technique for fully autonomous and efficient 3D robotics hand/eye calibration," IEEE Trans. Robot. Autom., vol. 5, no. 3, pp. 345-358, Jun. 1989.

[13] H. Zhuang, Z. S. Roth, and R. Sudhakar, "Simultaneous robot/world and tool/flange calibration by solving homogeneous transformation equations of the form AX = YB," IEEE Trans. Robot. Autom., vol. 10, no. 4 , pp. 549-554, Aug. 1994.

[14] A. Li, L. Wang, and D. Wu, "Simultaneous robot-world and hand-eye calibration using dual-quaternions and Kronecker product," Int. J. Phys. Sci., vol. 5, no. 10, pp. 1530-1536, Sep. 2010.

[15] G. D. van Albada, J. M. Lagerberg, A. Visser, and L. O. Hertzberger, "A low-cost pose-measuring system for robot calibration," Robot. Autom. Syst., vol. 15, no. 3, pp. 207-227, Aug. 1995.

[16] K. H. Strobl and G. Hirzinger, "More accurate camera and hand-eye calibrations with unknown grid pattern dimensions," in Proc. IEEE Int. Conf. Robot. Autom., Pasadena, CA, May 2008, pp. 1398-1405.

[17] S. D. Ma, "A self-calibration technique for active vision systems," IEEE Trans. Robot. Autom., vol. 12, no. 1, pp. 114-120, Feb. 1996.

[18] G. Q. Wei, K. Arbter, and G. Hirzinger, "Active self-calibration of robotic eyes and hand-eye relationships with model identification," IEEE Trans. Robot. Autom., vol. 14, no. 1, pp. 158-166, Feb. 1998.

[19] N. Andreff, R. Horaud, and B. Espiau, "Robot hand-eye calibration using structure-from-motion," Int. J. Robot. Res., vol. 20, no. 3, pp. 228-248, Mar. 2001.

[20] H. Malm and A. Heyden, "Self-calibration from image derivatives for active vision systems," in Proc. Int. Conf. Control Autom. Rob. Vision, vol. 2, Singapore, Dec. 2002, pp. 1116-1121.

[21] H. Malm and A. Heyden, "Self-calibration from image derivatives for active vision systems," in Proc. Int. Conf. Control Autom. Robot. Vision, vol. 2, Singapore, Dec. 2002, pp. 1116-1121.

[22] C. C. Wang, "Extrinsic calibration of a vision sensor mounted on a robot," IEEE Trans. Rob. Autom., vol. 8, no. 2, pp. 161-175, Apr. 1992.

[23] X. Haixia, W. Yaonan, C. Wei, and L. Juan, "A self-calibration approach to hand-eye relation using a single point," in Proc. Int. Conf. Inf. Autom., Zhangjiajie, China, Jun. 2008, pp. 413-418.

[24] A. Jordt, N. T. Siebel, and G. Sommer, "Automatic high-precision selfcalibration of camera-robot systems," in Proc. IEEE Int. Conf. Robot. Autom., Kobe, Japan, May 2009, pp. 1244-1249.

[25] S. D. Ma, "A self-calibration technique for active vision systems," IEEE Trans. Robot. Autom., vol. 12, no. 1, pp. 114-120, Feb. 1996.

[26] M. Staniak and C. Zieliński, "Structures for visual servos," Robot. Autom. Syst., vol. 58, no. 8, pp. 940-945, Aug. 2010.

[27] F. Dornaika and R. Horaud, "Simultaneous robot-world and hand-eye calibration," IEEE Trans. Robot. Autom., vol. 14, no. 4, pp. 617-622, Aug. 1998.

[28] J. S. Hu, M. C. Chien, Y. J. Chang, Y. C. Chang, S. H. Su, J. J. Yang, and C. Y. Kai, "A robotic ball catcher with embedded visual servo processor," in Proc. IEEE/RSJ Int. Conf. Intell. Robot. Syst., Taipei, Taiwan, Oct. 2010, pp. 2513-2514.
[29] L. Sun, J. Liu, W. Sun, S. Wu, and X. Huang, "Geometry-based robot calibration method," in Proc. IEEE Int. Conf. Robot. Autom., vol. 2, New Orleans, LA, Apr./May 2004, pp. 1907-1912.

[30] J. S. Hu and Y. J. Chang, "Calibration of an eye-to-hand system using a laser pointer on hand and planar constraints," in Proc. IEEE Int. Conf. Robot. Autom., Shanghai, China, May 2011, pp. 982-987.

[31] J. S. Hu and Y. J. Chang, "Eye-hand-workspace calibration using laser pointer projection on plane surface," Ind. Robot., Int. J., vol. 39, no. 2, pp. 197-207, 2012.

[32] D. C. Brown, "Close-range camera calibration," Photogramm. Eng., vol. 37 , no. 8, pp. 855-866, 1971.

[33] R. Willson, "Modeling and calibration of automated zoom lenses," $\mathrm{Ph} . D$. dissertation, Dept. Electr. Comput. Eng., Carnegie Mellon Univ., Pittsburgh, PA, Jan. 1994.

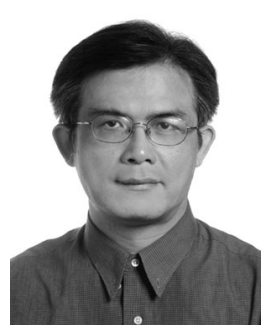

Jwu-Sheng Hu (M'94) received the B.S. degree from the Department of Mechanical Engineering, National Taiwan University, Taipei, Taiwan, in 1984, and the M.S. and Ph.D. degrees from the Department of Mechanical Engineering, University of California at Berkeley, in 1988 and 1990, respectively.

From 1991 to 1993, he was an Assistant Professor in the Department of Mechanical Engineering, Wayne State University, Detroit, MI. In 1993, he joined the Department of Electrical Engineering, National Chiao Tung University, Hsinchu, Taiwan, where he became a full Professor in 1998. He has served as the Vice Chairman of the Department since 2006. Since 2008, he has been working in part at the Industrial Technology Research Institute, Hsinchu, where he serves as the Advisor for the Intelligent Robotics program and the Principle Investigator of large-scale robotics research projects funded by the Ministry of Economic Affairs. He also serves as an Advisor at the National Chip Implementation Center of Taiwan for embedded system design applications. His current research interests include robotics, mechatronics, and embedded systems.

Dr. Hu and his laboratory developed a glove puppet robot system and received the 2008 IEEE IROS Best Entertainment Robot Award sponsored by the New Technology Foundation of Japan. He also received a Research Initiation Award from the National Science Foundation.

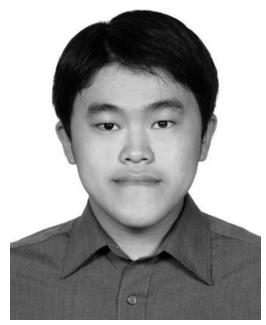

Yung-Jung Chang received the B.S. degree from the Department of Electrical Engineering, Yuan-Ze University, Jhongli City, Taiwan, and the M.S. degree from the Department of Control Engineering, National Chiao-Tung University (NCTU), Hsinchu, Taiwan, in 2005 and 2006, respectively. He is currently working toward the Ph.D. degree at NCTU and at the Industrial Technology Research Institute, Hsinchu.

His research interests include mobile robot localsensor fusion, and manipulator coordination. 